Carlos Hallet, S.J.

Profesor de la Universidad Católica del Norte

Antofagasta - Chile

\title{
El Tratado De Dilectione Dei de Balduino de Ford
}

\section{EL AUTOR}

Balduino de Ford o de Canterbury nació en el seno de una familia pobre del Devonshire, en una fecha desconocida. Gracias al apoyo de su obispo pudo estudiar en la escuela catedralicia de Exeter, su ciudad natal. Joven sacerdote diocesano, su reputación llegó hasta el papa Eugenio III, quien lo escogió como tutor de Graciano. Después de su estadía en el continente, fue profesor en Exeter y, en 1161, designado archidiácono de Totnes, en el Devon.

Ahora bien, su vocación era otra. En 1169 ingresó a la abadía de Ford recientemente fundada. En 1175 fue elegido abad, pero, en 1181, fue ordenado obispo de Worchester-on-Severn y, en 1184, nombrado arzobispo de Canterbury y primado de Inglaterra, lo que le valió enfrentar situaciones muy conflictivas con escaso éxito. En 1187, como legado papal, hizo la visita canónica del país de Gales. Al conocer la caída de Jerusalén en manos del sultán Saladino, se adhirió a la Tercera Cruzada y, en 1189, partió con Ricardo Corazón de León a Palestina. Apesadumbrado por la guerra y los excesos de los cruzados, murió en Tiro el 19 de noviembre 1190. (1)

De pequeña estatura, era un hombre amable, suave, moderado, sobrio, prudente, silencioso y siempre bien dispuesto al mismo tiempo que muy elocuente y docto.

\section{SUS OBRAS}

Autor de varios tratados y sermones, probablemente redactados durante su estadía en Ford, Balduino es un eminente exponente de la teología monástica, la que se considera como la continuación de la patrística.

(1) Balduino empieza a aparecer en las enciclopedias y diccionarios a comienzos del siglo XX. Ver F. AVELING, art. Baldwin of Canterbury, The Catholic Enciclopedia, vol. II, London, 1907, 221; Dictionary of National Biography, t. I, London, 1908, 952-954; J.M. CANIVET, art. Baudouin de Ford, Dictionnaire de Spiritualité, t. I, Paris, 1937, 1285. 
Sus escritos, esencialmente alimentados por la Sagrada Escritura y san Agustín, revelan también un buen conocimiento de los autores de la Antigüedad pagana.

El abad de Ford es un típico hijo del siglo XII, época de gran renovación intelectual y espiritual, que R. Javelet ha llamado "el siglo de oro de la contemplación" (2). Es un autor muy original, como ya lo señaló Dom Jean Leclercq, cuya teología está llena de espiritualidad a semejanza de la de san Bernardo (1090-1153) (3). Su pluma es más bien sucinta, pero penetrante y no desdeña los juegos de palabras para ser más convincente, invitar a la conversión e impulsar hacia la perfección.

Se conocen de él dos grandes tratados: el De sacramento altaris (4) y el De commendatione fidei (5), diversos tratados pequeños y sermones (6), algunas cartas (7), además de una docena de obras inéditas o extraviadas (8).

\section{EL TRATADO DE DILECTIONE DEI}

En otras oportunidades hemos descrito la dinámica de algunos temas recurrentes en la obra de Balduino (9) y hemos dedicado varias páginas a su famoso tratado "Sobre la vida cenobítica o común" (10).

Ahora presentamos otro tratado, pequeño, pero denso, claro y estimulante, que contiene unos análisis muy finos sobre las diferentes formas, insuficientes o perfec-

(2) R. JAVELET, Psychologie des auteurs spirituels du 12e siècle, Revue des Sciences Religieuses, 1959, p. 18.

(3) J. LECLERCQ-F. VANDENBROUCKE-L. BOUYER, La spiritualité du Moyen Age, MCMLXI, Aubier, p. 268

(4) Cf. MIGNE, Patrologia Latina, t. 204, 641-1674; Sources Chrétiennes, 93-94, Paris, Cerf, 1963, 595 p.; R.THOMAS, O.C.S.O., Baudouin de Ford, Pain de Cîteaux, 35, Chimay, Belgique, 1973, pp. 26-80. Traducción castellana por Elcira G.R. de SESMA, Cuadernos Monásticos 76 (1986), pp. 115-137.

(5) Cf. MIGNE, Patrologia Latina, t. 204, 571-640. Ver también Cîteaux, 1985, t. 36.

(6) Cf. MIGNE, t. 204, 403-774; R. THOMAS, Pain de Cîteaux, 35-45; Spiritual Tractates, 2 v., Kalamazoo, Michigan, Cistercian Publications, 1986, 226 p.; B.J.SAMAIN, Deux traités inédits de Baudouin de Ford, le "De oboedientia " et le "De sancta cruce", Cîteaux, 1988, fasc. 1-2, pp. 5-42.

Ver también: Opera: Sermones, De Commendatione Fidei, editado por David N. BELL, Brepols, Turnhout, Belgium, 1991, 502 p.

(7) MIGNE, Patrología Latina, t. 202, 1533; Chronicals and Memorials, London, 1865, t. II.

(8) D.N.BELL, The "Corpus" of the works of Baldwin of Ford, Cîteaux, 1984, fasc. 3-4, pp. $215-234$.

(9) C. Hallet, Temas antropológicos y vida espiritual según Balduino de Ford, Studia monastica, Abadía de Montserrat (Barcelona), vol. 29, 1987, fasc. 2, pp. 225-250; Le coeur et la vie spirituelle dans l'oeuvre de Baudouin de Ford, Studia monastica, Abadía de Montserrat (Barcelona), vol. 34, 1992, fasc. 2, pp. 305-314; El amor en la obra de Balduino de Ford, Studia monastica, Abadía de Montserrat (Barcelona), vol. 40, 1998, fasc. 2, pp. 281-290.

(10) C.Hallet, La communion des personnes d'après une oeuvre de Baudouin de Ford, Revue d'ascétique et de mystique, Toulouse, tome XLII, (1966), 4, nº 168, pp. 405-422; Notes sur le vocabulaire du "De vita coenobitica seu communi" de Baudouin de Ford, Analecta cisterciensia, Roma, annus XXII, 1966, fasc. 2, jul.-dec., pp. 272-278; Comunión y vida común, según Balduino de Ford. Homenaje al Profesor Dr. Julio Jiménez Berguecio, S.J., Anales de la Facultad de Teología, vol. XXXIII (1982), Universidad Católica de Chile, Santiago, 1983, pp. 159-169. 
tas, del amor de Dios y de la obediencia que le debemos (G. Jonquières S.J.). Se trata del De dilectione Dei (11).

\section{A. Visión de conjunto}

\section{1. "Amarás al Señor tu Dios"}

La insistencia repetitiva contenida en el primer mandamiento: “Amarás al Señor tu Dios con todo tu corazón, con toda tu alma, con todas tus fuerzas y con toda tu mente" (Mateo, 22, 37) llama la atención de Balduino, que ve en ella una intención divina.

Le parece que esa repetición puede ser comparada con unos martillazos. Así como para introducir un clavo en una madera muy seca hace falta golpearlo repetidas veces, de la misma manera para que entre el mandamiento en la dureza de nuestros corazones el Señor tiene que insistir reiteradamente hasta poder hacerlo penetrar en las últimas profundidades de nuestro ser.

Esta insistencia exagerada en imponer, indica también el deseo de Dios que cumplamos en forma desmedida.

Otra explicación de la cuadripartita distribución de este mandamiento, según Balduino, podría ser que Dios quiere darnos a entender que todas las células de nuestro corazón, hasta las más remotas, deben ser liberadas del amor del mundo para poder ser totalmente entregadas al amor de Dios.

\section{En este mundo Dios no es amado perfectamente}

Así como en la condición actual no conocemos a Dios perfectamente, tampoco lo amamos a la perfección. Estamos muy lejos del conocimiento y del amor que serán nuestros cuando veamos a Dios en su gloria.

Para Balduino, nuestro actual conocimiento de Dios es como la luz de la aurora que penetra en la casa por una pequeñísima ranura mientras que el del cielo será como el esplendor de toda la fuerza del sol al mediodía; y nuestro amor no es más que una diminuta chispa en comparación con el inmenso incendio que abrasará a los justos en la Jerusalén celeste.

Pero Dios nos pide que lo amemos en este mundo. La dificultad proviene del hecho que no adoramos a Dios con todo el corazón, porque no somos dueños de él: se nos escapa continuamente. Esta debilidad nos impide amar a Dios perfectamente con todo el corazón. Amémoslo, por lo menos, nos exhorta Balduino, en cuanto seamos capaces, es decir en la medida en que seamos dueños de nuestro corazón. No podemos imitar al que se niega a devolver parte de lo que debe, bajo el pretexto que no puede pagar toda su deuda de una vez. Pero Dios es un acreedor lleno de bondad. Al que le paga lo que puede, Él, en su misericordia, le da los medios para poder pagar más.

(11) Las referencias son de la edición de Migne, P.L., t. 204. 


\section{Dios debe ser amado con todo el corazón en sus beneficios}

En las cuatro partes del mandamiento, Balduino descubre la insinuación de que el amor tiene cuatro inclinaciones, afectos o modos principales que están relacionados con cuatro clases de realidades. Hay que amar a Dios con todo el corazón en sus beneficios, con toda el alma en sus promesas, con todas sus fuerzas en sus juicios y con toda la mente en sus preceptos.

El abad de Ford enumera varios beneficios divinos. En primer lugar, aquellos que corresponden a la creación del hombre: todo lo que somos, alma, cuerpo, sentidos del alma y del cuerpo, el ser de la raza divina, el tener en Él la vida, el movimiento y el ser (cf. Hechos, 17, 28) y el haber sido creados a su imagen y semejanza. Aquellos que conciernen nuestra "reparación": los misterios de la encarnación y de la pasión de Cristo, todos los sacramentos y sacramentales que nos remiten los pecados y nos procuran la salvación. Aquellos de cada día: consolaciones cotidianas, alivios de todos nuestros sufrimientos, auxilios para todas nuestras necesidades, remedios a todas nuestras dolencias como también deseos, peticiones y esperanzas.

Dios nos colma de beneficios, tratando así de conseguir nuestro amor y es muy justo que lo amemos con todo el corazón. Además, Él nos lo pide: "Dame tu corazón" (Proverbios, 23, 26). Quiere que se lo demos libre de todo pecado y vanidad para que se complazca en Él más que en cualquier otra realidad.

¿En qué consiste ese amor de Dios? Balduino explica que empieza por una conformidad que nos hace partícipes de un odio y de un desprecio común. Para amar a Dios con el corazón tenemos que estar concordes con Él en el odio y desprecio del mal. También lo amamos con el corazón si recordamos los bienes que recibimos de Él y los males que cometimos contra Él. Agradeciéndole, por una parte, y por otra haciendo penitencia, nos reconciliamos con Él y, dejando la discordia, volvemos a la concordia. Para Balduino es el primer grado del amor: la conversión del corazón, del mal al bien, de la vanidad a la verdad, de lo que desagrada a Dios a lo que le agrada. Amamos a Dios con todo el corazón, aunque imperfectamente, si detestamos con todo nuestro corazón el mal que Él odia.

\section{Dios debe ser amado con toda el alma en sus promesas}

Lo que Dios nos ha prometido es mayor que lo que Él nos ha dado hasta ahora.

Sus promesas son inmensas, inestimables. Prometió hacernos pasar del trabajo al descanso, de la esclavitud a la libertad, del temor a la seguridad, de la tristeza a la consolación, de la muerte a la resurrección y, en la resurrección, nos prometió una alegría plena, suma y sin deficiencia. Por fin, prometió darse a Sí mismo.

Por estas promesas y en ellas nos pide un cierto amor. ¿Qué clase de amor? Un amor que es deseo de las promesas, vehemente, fuera de medida, ya que las promesas de Dios superan todo deseo. Nuestra debilidad está siempre por debajo de lo que debería, nuestros deseos quedan cortos frente a lo que supera todo deseo, por eso el santo deseo, si tiene medida en lo que puede, no la tiene en lo que debe. Cuanto más crece y tanto más debe y nunca se va a exceder: puede y debe ser sin medida.

Por eso, en este caso de la espera de la promesa divina, la impaciencia, aunque vehemente, lejos de ser un pecado, es loable, ya que es signo de un mayor deseo, de 
un mayor amor. San Pablo conoció ese deseo y esa impaciencia (cf. Filipenses, 1, 23), pero nos dice que por la paciencia es posible esperar (Romanos, 8, 25), lo que significa que la impaciencia del santo deseo vehemente es paciente a pesar de los tormentos de la espera. Los justos son pacientes, incansables y valientes, soportan todas las adversidades y mantienen una esperanza inquebrantable.

\section{Dios debe ser amado con todas las fuerzas en sus juicios}

Balduino considera varios casos de juicios divinos. Aquí, su teología, marcada por la mentalidad de la época y una comprensión literal de la Biblia, es severa y presenta incluso a un Dios aparentemente poco leal.

Primero, está el juicio que ya ha condenado a los que están en el infierno. Después, el juicio muy misterioso por el cual Dios, escondiendo su ira como bajo la apariencia de la misericordia, deja que los malos realicen sus proyectos y, acumulando pecados, se pierdan. Otros malos reciben de Dios castigos en este mundo, pero en vez de purificarlos, esas pruebas provocan sus blasfemias: no aceptan la corrección y su impaciencia los lleva de los suplicios de la tierra a los eternos.

Por el contrario, los buenos alaban a Dios con motivo de los flagelos o aman los flagelos por Dios. Algunos, pacientes en las pruebas, aman a Dios en sus juicios, algunos otros se alegran en las pruebas y reciben los juicios divinos como beneficios. Existen otros que aman tanto los juicios de Dios que, sin esperar la corrección paterna, se enjuician y castigan a sí mismos. Estos, a la vez acusados, jueces y verdugos, odian su alma y así la guardan para la vida eterna (cf. Juan, 12, 25).

En sus juicios, Dios prueba a sus elegidos, los educa a la paciencia, los ejerce para que sean capaces de soportar los sufrimientos y los conforta por la esperanza (cf. Romanos, 5, 3-4). La certeza que da la esperanza y la magnitud de la gloria esperada alivian el peso de la tribulación y hasta consiguen que se la ame.

Para el alma que se deleita en los juicios de Dios, los sufrimientos son poca cosa y la multiplicidad de las tentaciones una alegría.

\section{Dios debe ser amado con toda la mente en sus preceptos}

La mente en el hombre es aquello que organiza, manda y debe cuidar de ser obedecido, pero debe acordarse de que tiene la obligación de obedecer a Dios. La obediencia que ella exige de sus inferiores, es justo que la deba a su superior. Por otra parte, para ella, desobedecer sería la muerte, es decir lo peor, lo más terrible. Ahora bien, si la desobediencia es tan abominable como la muerte que ella causa, ¿no se puede pensar que la obediencia, que está unida a la vida, es tan digna de amor como la misma vida?

Amor de Dios y obediencia son inseparables. Lo afirmó el mismo Señor, cuando dice: "Quien me ama guarda mi palabra" (Juan, 14, 23) y "Aquel que no me ama, no guarda mis mandamientos" (Juan, 14, 24). No hay caridad sin obediencia, ni obediencia sin caridad. Quien ama a Dios de verdad, ama también su mandamiento. Aquel que ama la voluntad de Dios y sus mandamientos, ama también la obediencia, la cual no puede existir sin amor de la obediencia y amor del mandamiento. 
Existen tres maneras de amar los mandamientos: meditándolos, poniéndolos en práctica sin pereza y ejerciéndolos en las pruebas.

En cuanto a la obediencia, hay que distinguir dos clases: la verdadera y la fingida. Desde el punto de vista del sentimiento interior se distinguen también dos clases: la obediencia forzada y aquella que es realmente voluntaria. La forzada es conseguida por la fuerza del mandamiento, la segunda nace en forma espontánea por la presión del amor.

La obediencia puede ser al mismo tiempo forzada y verdadera: es una obediencia con más temor que amor. Pero si es verdadera y espontánea, en ese caso, domina el amor, aunque siempre está presente algo de temor.

La obediencia puede ser también forzada y fingida. En este caso contiene solo temor y no está orientada hacia Dios.

Existe además una obediencia voluntaria y al mismo tiempo fingida, como la de los hipócritas. En cuanto a la obediencia voluntaria y verdadera, procede de la caridad y tiende hacia Dios por la caridad. Es aquella que Dios ama y recompensa.

Balduino hace notar que la obediencia forzada que es verdadera no es reprobable, ya que el temor del castigo le impide pecar. Ella se ordena hacia Dios, a quien honra, y por eso merece una cierta recompensa. Sin embargo, Dios tiene derecho a una obediencia voluntaria y verdadera.

Balduino precisa: obedecer a Dios voluntaria y verdaderamente supone que la criatura quiera lo que Él quiere porque Él lo quiere, e incluso que ella quiera que Él quiera lo que quiere. Esto se aplica en particular al amor de los enemigos: aunque estoy dispuesto a hacer el bien a mis enemigos, si me gustara que Dios no hubiera mandado ese amor, no quiero todavía plenamente lo que Dios manda. En este caso, le falta a mi obediencia lo que precisamente falta a mi caridad: el ser feliz de hacer lo que a Dios le agrada porque le agrada.

Balduino termina este párrafo y el tratado recordándonos que obediencia y caridad tienen un solo fin: agradar a Dios en lo que le agrada.

Esta última precisión la coloca Balduino, porque constata que hay hombres malos que quieren agradar a Dios en lo que a ellos les agrada con la idea de conseguir sus beneficios y promesas. De hecho no agradan a Dios, porque su caridad no está ligada a la obediencia, sino simplemente a la opinión que ellos se hacen de lo que es la sabiduría. Ahora bien, la obediencia, que es la compañera de la caridad, se apoya siempre en la voluntad de Dios: la voluntad divina es el principio y el fin de la obediencia. La obediencia de los malos es una obediencia sin amor: ella no puede agradar a Dios.

\section{B. Inspiración bíblica}

La profunda inspiración bíblica de la vida y del pensamiento de Balduino se refleja en este tratado por el gran número de citas tanto de la Antigua como de la Nueva Alianza.

Si Balduino acude al Deuteronomio, al Segundo Libro de Samuel, a Isaías (2 veces), a Daniel y al Cantar de los Cantares, es en los autores sapienciales donde encuentra su mayor apoyo: el libro de Job, el Eclesiastés, el Eclesiástico y los Proverbios (3 veces). Pero su referencia constante son los Salmos. Cita 22 de ellos 
una vez; a otros 3, dos veces y 9 veces al más largo de todos, el salmo 119 (118) cuyo leitmotiv está muy relacionado con el tema principal del De dilectione Dei, ya que es un elogio de la Ley, fuente de alegría, consuelo, felicidad, provecho, sabiduría, salvación, seguridad y vida.

La Nueva Alianza está presente a través de los evangelios de Mateo (4 veces), Lucas y Juan (6 veces), los Hechos de los Apóstoles, la Carta a los Romanos (4 veces), la Primera y la Segunda Carta a los Corintios (cada una, 2 veces), las Cartas a los Filipenses (3 veces) y a los Hebreos, la Epístola de Santiago y el Apocalipsis (2 veces).

\section{La retórica al servicio de la teología monástica}

Hijo de una cultura latina marcada por Cicerón y Agustín, el abad de Ford, famoso por su elocuencia, ha desplegado sus mismas cualidades de orador en sus escritos. Lo que nos explica, por ejemplo, la frecuencia de enumeraciones casi pleonásticas. No faltan las de dos términos, como las siguientes: "exaggeratio, inculcatio" (417 D); "transfigere et transpenetrare" (419, B); "imperfecte et non plene" (419, D); "tenere, retinere" (420, C); "Quantum debes, quantum teneris" (420, D); "magnae... et inaestimabiles" (422, D); "glorificant et laudant" (424 C); "firmiorem et certiorem" (425, B); "summum et praecipuum" (425 C); "mandata Dei servare et Deo oboedire" (427, B); "summa et principali" (429, A).

Balduino multiplica las enumeraciones. Las hay de tres términos, algunas tomadas directamente de los textos paulinos. De la Carta a los Hebreos $(4,12)$, al hablar de la palabra de Dios: "vivus, et efficax, et penetrabilior" (419, B); de los Hechos de los Apóstoles $(17,28)$ : "in ipso vivimus, movemur et sumus" (421, B). Las demás son del mismo Balduino: "omnes cogitationes, omnes affectiones, omnes quoque intentiones" $(420, \mathrm{C})$; "quantum potes, quantum praevales, quantum sufficis" (420, D); "alia beneficia conditionis, alia reparationis, alia quotidianae consolationis" (421, B); "desiderare, petere et sperare" (421, C); "omnium dolorum..., omnium necesitatum..., omnium infirmitatum" (ibid.); "et merito, et digne, et justissime" (421, D); "quam salubria sunt, quam honesta, quam fidelia" (426, D); "vel tentationis, vel persecutionis, vel adversitatis" (427 A); "meditationis, et actionis, et exercitationis" (ibid.); "qui non fornicantur, qui non furantur, qui...non persequuntur" $(427$, B); "quae mandat, et cui mandat, et quam mandat" (430, A).

Otras son cuádruples: "tam diligens, tam exquisita, tam exacta, tam propensa" (417, D); "probat..., et erudit..., et exercet..., et confortat" (425, A); "dispensat, ...ordinat, ...imperat, ...judicat..." (425 D). Hay hasta una quíntuple: "avolat, revolat, excurrit, discurrit et recurrit" (420, B).

Aliteraciones y consonancias como estas últimas son frecuentes bajo la pluma del autor del "De dilectione Dei": "...corde..., concordes... Corde..., recordantes, ...discordiam... concordiam" (422 A); "desiderat et suspirat, donec in illo respiret, ad quod aspirat" (423, C); "qui in sordibus sunt, sordescant adhuc" (424, A); "justitiae norma, et aequitatis forma" (429, A). Es muy significativa también la última frase del tratado: "Qui enim in caritate obedit, quasi ad finem ad illum suspirat, qui $\mathrm{ab}$ initio inspirat; in quo inspiratus et suspirans a labore quem fingit in praecepto sine fine respirat" (430 A-B). 
No solamente Balduino no teme las repeticiones, sino que les tiene una gran afición. Es así como en una frase de veinte palabras, usa 4 veces "flagella" y le añade "flagellare" (424 C); en otra de una treintena de vocablos, emplea "reus" 4 veces, "judex" 3 veces, "juste" 2 veces, "justus" 1 vez y "judicare" 1 vez (425 A). Logra repetir "Deus" 13 veces y "voluntas" 9 veces en un texto que merece ser citado por entero:

"Oboedientia itaque tota in dilectione Dei constituitur; quia a voluntate Dei per voluntatem Dei ad voluntatem Dei tendit, et ad voluntatem Dei redit. Voluntas enim Dei in Deo per voluntatem Dei in homine operatur voluntatem Dei in mandato, propter voluntatem Dei in Deo. Ergo voluntas Dei in Deo principium est et finis oboedientiae". (430, A).

Y en un párrafo decisivo, aunque corto, sobre el amor y la obediencia, repite "oboedientia" 7 veces -además de "oboedire" 2 veces- y "amor" 12 veces, además de "amare" 4 veces (426 C).

El tratado está también repleto de oposiciones bipolares. De substantivos: "amor mundi", "amor Dei" (419 C); "in praesenti", "in gloria Dei" (419 D); "servi", "filii" (421 C); "discordia", "concordia" (422 C); "malum", "bonum" (422 B); "vanitas", "veritas" (ibid.); "labor", "requies" (422 C); "servitus", "libertas" (ibid.); "timor", "securitas" (ibid); "moeror", "consolatio" (ibid.); "mors", "resurrectio" (ibid.); "misericordia", "ira" (424 A); "mali", "boni" (424 C); "inferior", "superior" (425 D); "inoboedientia", "oboedientia" (426 A); "meditatione et actione", "exercitatione" (427 A); "timor", "amor" (427 D); "principium", "finis" (430 A).

De verbos: "displicere", "placere" (422 B); "complectuntur", "disjunguntur" (426 A); "procedere", "tendere" (427 D). De adjetivos: "patiens", "impatiens" (423 B); "odibilis", "diligenda" (426 A); "dulcia", "amara" (426 D); "suavia", "gravia" (ibid.); "vera", "simulata" (427 C); "coacta", "voluntaria" (ibid.); "improbanda", "approbanda" (428 A); "volens", "nolens" (428 C) y de adverbios: "imperfecte" (419 C), "perfecte" (420 C); "patienter" (423 A), "impatienter" (423 B).

Si Balduino no usa mucho el quiasmo (ver, sin embargo, en 427 D: "dulcia... et suavia, ...gravia ...et amara"), le gusta de vez en cuando emplear alguna metáfora. Es así como -además de una que otra sacada de la Biblia- hace intervenir en su texto algunas comparaciones tomadas de la naturaleza: la hoja de un árbol (419 A) y el forraje (424 B), también la luz de la aurora que penetra en la casa por una pequeña rendija (419 D) y el esplendor del sol al mediodía (ibid.), una pequeña centella y un gran incendio (420 A). Recurre también al trabajo necesario para introducir un clavo en una madera muy dura (419 B) y a los humanos: un deudor inmoral que no paga lo que podría bajo el pretexto que no puede solventar el conjunto de su deuda (420 D) y el padre de familia que organiza su casa y manda a todos (425 C-D).

\section{Algunos grandes temas}

Los párrafos siguientes son una presentación de algunos temas más importantes. En ellos se encuentran más de una idea ya expresadas en la "Visión de conjunto", pero que estimamos no podían ser omitidas. 


\section{Dios}

En el tratado De dilectione Dei, Dios, para Balduino, es el Señor Dios, que hay que amar con todo el corazón, toda el alma, todas las fuerzas y toda la mente (cf. Mateo, 22, 37).

Es también el Padre omnipotente y "providente" (419 A), que se ha preocupado por dejarnos por escrito el mandamiento del amor divino.

El Creador (428 B) es un acreedor lleno de bondad y misericordia (420 d). Todos nuestros bienes no son más que dones de Él (421 B), Padre de las misericordias y Dios de todo consuelo (II Corintios, 1, 3-4), que nos consuela a diario en todas nuestras pruebas $(421 \mathrm{C})$. Él es el inspirador de nuestros deseos, nuestras peticiones y nuestra esperanza. Es de Él que podemos esperar el alivio, el auxilio y el remedio de todos nuestros males (ibid.). La multitud de sus beneficios nos aplasta y debemos amarlo con todo el corazón (421 D sqq). En cuanto a sus promesas sobrepasan todo deseo (422 C sqq). Sus juicios son misteriosos (423 D- 424 A), pero justificados (425 B; cf. Salmo 18, 11) y fuentes de felicidad (425 C).

Lo que Dios, el Altísimo (423 C), ama y recompensa es la obediencia voluntaria y auténtica (427 D) a su voluntad, que es la norma primera y suprema de la justicia (429 A).

Cristo interviene poco en este tratado. Él es la persona que nos redime por los misterios de la Encarnación y de la Pasión, y con todos los sacramentos y sacramentales, a los cuales Dios, en su misericordia, ha dado la gracia de procurar la salvación de los creyentes (421 B-C).

El Espíritu Santo, por su parte, es el que sopla en el alma para fomentar sus deseos $(423 \mathrm{C})$.

\section{Los hombres}

Balduino distingue los creyentes, indignos servidores que Dios, sin embargo ha dignado hacer hijos suyos (421 C), los justos (423 B), los santos (423 C) y los elegidos (423 D) de los reprobados y de aquellos que ya están condenados, colocados en el infierno, donde nadie ama a Dios e incluso donde nadie se ama a sí mismo (ibid.)

Identifica malos con enemigos de Dios (424 A). Algunos de ellos quieren agradar a Dios, pero en lo que a ellos les agrada y no se dan cuenta de que, al actuar así, le desagradan (428 B). Otros insensatos, pecadores, inicuos corren alegremente hacia su perdición (424 B). Otros son blasfemos, murmuradores e impacientes. Otros aceptan ser corregidos y no aman los juicios de Dios: pasarán de los sufrimientos temporales a los suplicios eternos (424 C).

Así como clasifica a los malos en diversas categorías, así también Balduino distingue varias clases de hombre buenos. Algunos, con paciencia y amor, glorifican y alaban a Dios con motivo de las pruebas. Otros se alegran en las dificultades de la vida, bendiciendo a Dios y celebrando su gloria en la adversidad, porque reconocen que son culpables y merecedores de castigos. Otros, en un exceso de amor, se corrigen a sí mismos, sin esperar que les sobrevenga la corrección (424 C-D).

A todos sus elegidos Dios les prueba, les forma a la paciencia y a la aceptación de los sufrimientos al mismo tiempo que les fortalece por la esperanza (425 A). 
El que ama a Dios por encima de todo se deleita al saborear sus juicios (425 C). El que le ama de verdad ama también sus mandamientos (426 B) y aquel que ama los mandamientos y la voluntad de Dios ama también la obediencia (426 C). Ahora bien, frente a los mandamientos, los hombres reaccionan en forma diversa. Algunos son buenos para meditar, pero perezosos para actuar; otros, frente a las pruebas, son pusilánimes e inconstantes (427 A); muchos observan los mandamientos, pero les gustaría más que no existieran y cumplen por temor al castigo: esos no aman la obediencia (427 B-C). Lo lógico, sin embargo, sería que la criatura razonable se someta a su Creador queriendo lo que Él quiere, porque lo quiere, e incluso queriendo que Él quiera lo que quiere (428 B).

\section{Mente, voluntad y corazón}

Más que al alma o al espíritu, Balduino da importancia a la mente, la cual ocupa, en el hombre, el lugar más elevado y de lejos el principal. Él la compara con un "paterfamilias" que ordena y organiza el conjunto de su casa según su criterio, único señor y juez, que exige obediencia en todo. Pero la mente, que exige tanto sometimiento, debe acordarse de que ella también debe obediencia a Dios y debe amarlo con todo lo que ella es (425 C-D).

En cuanto a la voluntad, para ser "informada" por la voluntad de Dios, ella debe querer lo que Dios quiere, porque Él lo quiere: así, se vuelve buena, porque Dios mismo pasa a ser su principio y causa. En caso contrario, la voluntad queda todavía mía (428 B), es decir, que no es capaz de amar a Dios perfectamente. La llaman "voluntad propia". Contra ella batalla la obediencia, compañera de la caridad (429 A).

La voluntad del hombre pasa a ser "voluntad de Dios" cuando recibe su "forma" de la norma suprema y primera de la justicia, que es la misma voluntad de Dios. Balduino recuerda que se llama "voluntad de Dios" a aquella que está en Dios, pero también a aquella que está en el hombre y que viene de Dios, no solo del hombre: es la voluntad de Dios presente en la persona que es mandada y obedece (429 A-430 A).

Balduino constata que el corazón de los hombres es duro (419 B) y que, por otra parte, no les pertenece: hecho pedazos, pertenece a otros y los hombres, abandonados por su propio corazón, están allí, como personas sin corazón (420 A-B).

Personalmente hace constantemente la experiencia que, incluso en la oración, su corazón es tan volátil como una mariposa. Está en todas partes y en ninguna y se escapa continuamente. Esto es señal de que todavía no ama a Dios tan perfectamente como Él quiere: con todo el corazón.

Mientras tanto hay que amar a Dios y adorarlo imperfectamente. Si se hace con sinceridad y si uno ofrece su corazón a Dios en la medida de sus fuerzas, Dios lo recibe y lo hace nuestro. Cuanto más uno entrega su corazón a Dios, tanto más el corazón se vuelve nuestro.

Amar al Señor perfectamente con todo el corazón significaría dirigir hacia Él todos los pensamientos, todos los afectos y todas las intenciones y mantenerlos en Él sin que se salgan de ese centro divino. Sería, además, arder interiormente, con todos sus meollos, del fuego del amor. Pero esto no está en el poder de la debilidad humana.

Ya que no podemos amar como deberíamos, tratemos, por lo menos, de hacerlo en cuanto somos capaces, con las fuerzas que tenemos y, poco a poco, amaremos 
más perfectamente con un corazón cada día más nuestro (420 A-D). Esto llegará a ser realidad a medida que lo entreguemos a Dios, que lo quiere entero, liberado del amor del mundo y de todo lo que es del mundo, centrado en Él, encontrando en Él todas nuestras complacencias más que en cualquier otra cosa, a tal punto que nos disguste lo que al Señor no le gusta (421 D).

Amamos a Dios "de corazón” si concordamos con Él en el odio del mal. Lo amamos si guardamos en el corazón los bienes que recibimos de Él para agradecérselos y los males para hacer penitencia y reconciliarnos con Él, saliendo de este modo de la discordia para volver a la concordia (422 A). Esta conversión del corazón, de la vanidad a la verdad, es el primer grado del amor (422 B).

\section{El amor}

Balduino opone al amor del mundo ("amor mundi"), que ocupa todas las células de nuestro corazón (419 C), al amor de Dios ("amor Dei", "amor divinus", "dilectio Dei", "caritas") (12), que es como un clavo con el cual el Señor quiere atravesar completamente ("transpenetrar") nuestro corazón (419 B). Ahora bien, el amor del mundo debe ser eyectado definitivamente del corazón para que Dios lo pueda poseer enteramente, hasta sus rincones más remotos, y que así él pueda también poseer a Dios (419 C-D).

Para que logremos la perfección del amor, el Señor multiplica sus beneficios y nos sostiene con sus promesas, sus juicios y sus preceptos (420 D; 421 A-D). El primer grado del amor de Dios hace que, por la conversión del corazón, el alma aborrezca el mal y opte por el bien (422 A-B), pero más allá de ese primer paso, ella empieza a amar a Dios con un deseo vehemente de sus promesas (422 D). Amando más a Dios, ella ama su voluntad y sus preceptos. Balduino insiste en que el amor auténtico es inseparable de la obediencia, cuyo amor precede el amor de los mandamientos (426 B-C): los hombres que no aman los preceptos, no aman la obediencia (427 A). Esta virtud, cuando es perfecta, es voluntaria y libre, ya que ella brota, con fuerza, de la violencia del amor (427 C).

Comparado con el gran incendio del amor que abrasará a los justos en la Jerusalén celestial, el amor de Dios en este mundo no es más que una chispa (420 A), pero va creciendo con la obediencia, si uno ofrece su corazón a Dios y hace todo lo posible por amarlo con toda el alma, con todas las fuerzas y con toda la mente. Al amar a Dios en sus beneficios, sus promesas, sus juicios y sus preceptos el alma se prepara a amarlo algún día plena y perfectamente en Sí mismo (421 A). Aumentan sus deseos (cf.422 D), junto con la paciencia, la esperanza (423 B) y la obediencia (426 A), la cual, guiada por la caridad, ve a Dios en el mandamiento, mientras que la caridad, guiada por el mandamiento, dirige la obediencia hacia Dios (426 B). El amor por el cual se ama a Dios crece íntimamente ligado al amor de su voluntad, de sus mandamientos y de la obediencia (426 C)).

El que ama los mandamientos lo hace por la meditación, el ejercicio y su práctica en las pruebas (426 D). Avanzando constantemente, el alma llega a una caridad y una obediencia más perfectas que la vuelven feliz de hacer lo que agrada a Dios (428 D).

(12) Sobre el vocabulario del amor en Balduino, ver C. Hallet, Notes sur le vocabulaire..., 1.c., pp. 274-276. 
Balduino identifica amor y obediencia. Amiga de la caridad, la obediencia consiste en el amor de Dios. En ese amor identificado con la obediencia, el obediente suspira hacia Aquel que lo inspira y en Quien respirará sin fin (cf. 430 A-B).

$$
* * *
$$

Dedicado al Primer Mandamiento, este tratado de Balduino demuestra la capacidad de su autor para elucidar las intenciones del corazón humano y para celebrar la elevadísima vocación del ser humano a la perfección del amor.

$\mathrm{Su}$ análisis realista de las exigencias fundamentales de la vida espiritual sigue siendo actual a pesar de los siglos y confirma a Balduino de Ford como a un destacado autor espiritual que merece no ser olvidado.

\section{RESUMEN}

Figura relevante de la Orden del Císter, Balduino de Ford es un autor que se distingue por la originalidad de su espiritualidad.

Su tratado De dilectione Dei es una meditación sobre el Primer Mandamiento, que nos invita a amar a Dios "con todo el corazón en sus beneficios, con toda el alma en sus promesas, con todas las fuerzas en sus juicios y con toda la mente en sus preceptos".

Su reflexión, nutrida de la Biblia y expresada en un lenguaje marcado por la retórica latina, insiste en que el amor perfecto exige del creyente amar lo que a Dios le agrada porque a Él le agrada. Termina identificando prácticamente amor con obediencia.

\footnotetext{
ABSTRACT

Famous figure of the cistercian order, Baldwin of Ford is an author who stands out for the originality of his spirituality.

His treatise "De dilectione Dei" is a meditation about the First Comandment, which is an invitation to love God "with all the heart in His benefits, with all the soul in His promises, with all the forces in His judgements and with all the mind in His precepts".

According to his reflection, fed with the Bible and expressed in a language marked by the latin rhetoric, the perfect love requires to love what is pleasing to God because it pleases Him. Baldwin practically identifies love with obedience.
} 